International Journal of Biological Research, $2(2)(2014) 121-124$
International Journal of Biological Research
JPC \begin{tabular}{c} 
Journal home page: $\begin{array}{c}\text { www.sciencepubco.com/index.php/IJBR } \\
\text { do.14419/ijbr.v2i2.3314 } \\
\text { Research Paper }\end{array}$ \\
\hline
\end{tabular}

\title{
A comparative evaluation of the inulin utilization potential of two Bifidobacterium animalis subsp. lactis strains for the development of a synbiotic powder
}

\author{
Ligimol James $^{1 *}$, Anil K.Puniya ${ }^{2}$, Kishan Singh ${ }^{2}$ \\ ${ }^{1}$ Department of Dairy Microbiology, College of Dairy Science and Technology, Mannuthy, Thrissur, India \\ ${ }^{2}$ Dairy Microbiology Division, National Dairy Research Institute, Karnal, Haryana \\ *Corresponding author E-mail: ligi11@ rediffmail.com
}

\begin{abstract}
Assessment of the compatibility between the probiotic and prebiotic is identified as a critical step while developing synbiotic products. So a study was conducted to compare the inulin utilization potential of two Bifidobacterium animalis subsp. lactis strains for selecting a strain for the development of a lyophilized synbiotic product. The growth stimulatory effect of inulin was found to be more prominent on Bifidobacterium animalis subsp. lactis B420 than on Bifidobacterium animalis subsp. lactis Bb-12. So a synbiotic pair of Bifidobacterium animalis subsp. lactis B420 and inulin was selected for the development of the synbiotic product. The developed product had a high probiotic count of $6.5 \times 10^{8} \mathrm{cfu} / \mathrm{g}$, showing a survival rate of $65 \%$. On assessing the probiotic count of the developed product during storage, a marked reduction was observed on room temperature storage with the counts reducing to the level of less than five $\log \mathrm{cfu} / \mathrm{g}$ after 60 days. In contrast the count remained in the range of eight to nine log cfu/g even after 60 days of refrigerated storage. So it could be a suggested that considering the probiotic stability and viability problems pertaining to fermented dairy products, lyophilized products could be potential alternatives as matrices for the delivery of probiotics to humans.
\end{abstract}

Keywords: Bifidobacterium animalis subsp. lactis Bb-12, Bifidobacterium animalis subsp. lactis B420, Inulin, Lyophilized powder, Synbiotics.

\section{Introduction}

Synbiotics, which exploit the synergistic relationship between its two constituents, the probiotics and prebiotics, are now gaining wide popularity as a means of altering the gut microbiota towards a beneficial one. It is presumed that the ready availability of a substrate that specifically stimulates the probiotic will enhance the survivability, colonization and beneficial effects of the probiotic thereby improving the effectiveness of the developed product. Although the synbiotic approach has attracted interest, the development of synbiotics is still at an early stage (Saulnier et al. 2008). Maintenance of viability of probiotics and selection of a synergistic prebiotic are considered as the major challenges for developing successful synbiotic products. The bacteria used as probiotics are generally selected from the genera Lactobacillus and Bifidobacterium. Among bifidobacteria, B. animalis is the most widely used species in foods due to its high tolerance to oxygen and acids (Ma"tto et al. 2004). B animalis subsp. lactis is a species evolved from the continuous adaptation of $B$ animalis to the fermented dairy environment (Palaria et al. 2012). The most widely documented probiotic Bifidobacterium strain, Bb-12 also belongs to this species. The prebiotic inulin is widely acclaimed for its bifiodogenic attributes and a synbiotic combination of this prebiotic with Bifidobacterium is well recognized as a synbiotic pair. However wide variations are observed among the members of the genus Bifidobacterium in their ability to utilize inulin. Inulin utilization is identified as a strain-specific feature among bifidobacteria (Rossi et al. 2005). Fermented dairy products like yoghurt are traditionally being used as delivery vectors for probiotics and prebiotics. It is estimated that $78 \%$ of current probiotic sales in the world are delivered through yoghurt (Granato et al. 2010). However the major constraints with these products are the maintenance of probiotic counts in required levels and the short shelf life of the product. Development of freeze dried synbiotic products could be a better option in this direction. Considering these aspects two commercially available probiotic Bifidobacterium animalis subsp. lactis cultures namely Bifidobacterium animalis subsp. lactis B420 and Bifidobacterium animalis subsp. lactis Bb-12 were assessed for their ability to utilize the prebiotic inulin to develop a lyophilized symbiotic preparation using the selected synbiotic pair.

\section{Materials and methods}

\subsection{Prebiotic and probiotic cultures}

Inulin was procured from Orafti, Belgium. The probiotic cultures, Bifidobacterium animalis subsp. lactis B420 (B-420, Danisco, Germany) and Bifidobacterium animalis subsp. lactis Bb-12 (Chr. Hansen, Denmark) were maintained in MRS broth supplemented with $0.05 \%$ L-cysteine hydrochloride (mMRS, Arroyo et al. 1994) with weekly subculturing. Purity of the culture was monitored periodically by plating on Bifidobacterium agar (HiMedia Laboratories Ltd., Mumbai). The culture was activated by 2 to 3 transfers in mMRS media with anaerobic incubation (using Anaero Hi Gas Pack, HiMedia Laboratories Ltd. Mumbai) at $37^{\circ} \mathrm{C}$ for $24 \mathrm{~h}$. 


\subsection{Inulin utilization potential of Bifidobacterium ani- malis subsp. lactis strains}

$24 \mathrm{~h}$ old active Bifidobacterium cultures $\left(10^{9} \mathrm{cfu} / \mathrm{ml}\right)$ were inoculated into a semi-liquid (agar $0.1 \%, \mathrm{w} / \mathrm{w}$ ) minimal media (meat peptone 1 percent $(\mathrm{w} / \mathrm{v})$, L-cysteine hydrochloride 0.04 percent (w/v), buffering salts and indispensable ions as in Garches medium (Rasic, 1990) containing different levels of inulin $(0,0.5,1,3$ and 5 percent (w/v)) (Bielecka et al. 2002). Bifidobacterial counts were determined at $0 \mathrm{~h}$ and $24 \mathrm{~h}$ using Bifidobacterium agar and the growth stimulatory effect of inulin was assessed based on the percentage reduction in mean generation time in inulin containing media compared to that in control tube ( $0 \%$ inulin).

2.3. Development of a freeze-dried (lyophilized) synbiotic preparation containing the selected Bifidobacterium strain and inulin and its storage study

The freeze-dried synbiotic preparation was developed based on the method of Collins and Hall (1984). For this, the selected culture was inoculated into mMRS broth @ 2 percent and incubated at $37^{\circ} \mathrm{C}$ for $48 \mathrm{~h}$ under anaerobic conditions. After this, the cells were harvested by centrifugation at $12000 \mathrm{rpm}$ for $10 \mathrm{~min}$ at $4^{\circ} \mathrm{C}$, washed once in sterilized distilled water and resuspended @ $10^{9}$ $\mathrm{cfu} / \mathrm{ml}$ in a sterilized mix containing 5 percent non-fat dry milk (NFDM), 20 percent inulin, 8 percent sucrose and 1.5 percent gelatin (gelatin was separately sterilized and mixed aseptically with the solution containing other components). The mix thus obtained was poured into sterile petri plates and freeze dried (Alpha 1-4 Freeze dryer; Martin Christ, Germany).

In order to carry out storage studies the synbiotic product was aseptically transferred to sterile moisture proof $150 \mathrm{ml}$ polypropylene bottles and stored at room and refrigeration temperatures. Enumeration of probiotic organisms was done by plating on Bifidobacterium agar on $0,7,14,21,30$ and 60 days of storage. Data was statistically analyzed using ANOVA according to the General Linear Models procedure of Systat Version 6.0.1 (1996, SPSS Inc.). When significant (1 and 5\% levels) differences were observed individual values were compared by Fisher's Least Significant difference.

\section{Results}

\subsection{Inulin utilization potential of Bifidobacterium ani- malis subsp. lactis strains}

The ability of Bifidobacterium animalis subsp. lactis B420 and Bifidobacterium animalis subsp. lactis Bb-12 to utilize, inulin was determined by monitoring their growth in a semi-liquid minimal media (Table 1). Both the strains could utilize inulin as a reduction in mean generation time compared to the control ( $0 \%$ inulin) was observed for both the Bifidobacterium strains. However differences were observed between the two strains in their inulin utilization pattern. The growth stimulatory effect of inulin was more prominent in the case of Bifidobacterium animalis subsp. lactis $\mathrm{B} 420$, as a marked reduction in its mean generation time was observed compared to that of Bifidobacterium animalis subsp. lactis Bb-12. Among the different concentrations of inulin used, supplementation at the rate of 0.5 percent showed a significantly lower growth stimulatory effect in comparison to other concentrations. However, no significant difference was observed among other inulin concentrations $(1,3$ and $5 \%)$ in terms of growth stimulatory effect $(\mathrm{P}<0.05)$.

Table 1: Utilization of Different Concentrations of Inulin (Mean Generation Time, Hours)

\begin{tabular}{llllll} 
Culture Name & \multicolumn{6}{l}{ Inulin Concentration } \\
\cline { 2 - 4 } & $0 \%$ & $0.5 \%$ & $1.0 \%$ & $3.0 \%$ & $5.0 \%$ \\
animalis subsp. lactis & 46. & 26.10 & 19.40 & 18.18 & 19.20 \\
Bb-12 $^{\mathrm{a}}$ & 3 & $(43.63$ & $(58.10$ & $(60.73$ & $(58.53$ \\
Bifidobacterium & & $\%)$ & $\%)$ & $\%)$ & $\%)$ \\
animalis subsp. lactis & 58. & 20.50 & 19.80 & 11.40 & 12.12 \\
B420 $^{\mathrm{b}}$ & 8 & $(65.11$ & $(66.32$ & $(80.61$ & $(79.39$ \\
\hline
\end{tabular}

$\mathrm{ab}$ - Bifidobacterium cultures bearing different superscripts differ significantly $(\mathrm{P}<0.05)$. Values in parenthesis are percent reduction in mean generation time compared to control ( $0 \%$ inulin).

\subsection{Development of the synbiotic powder and its storage studies}

As the growth stimulatory effect of inulin was more prominent on Bifidobacterium animalis subsp. lactis B-420, this culture was selected for the development of freeze dried synbiotic powder. The flowchart for the preparation is shown in Fig.1.

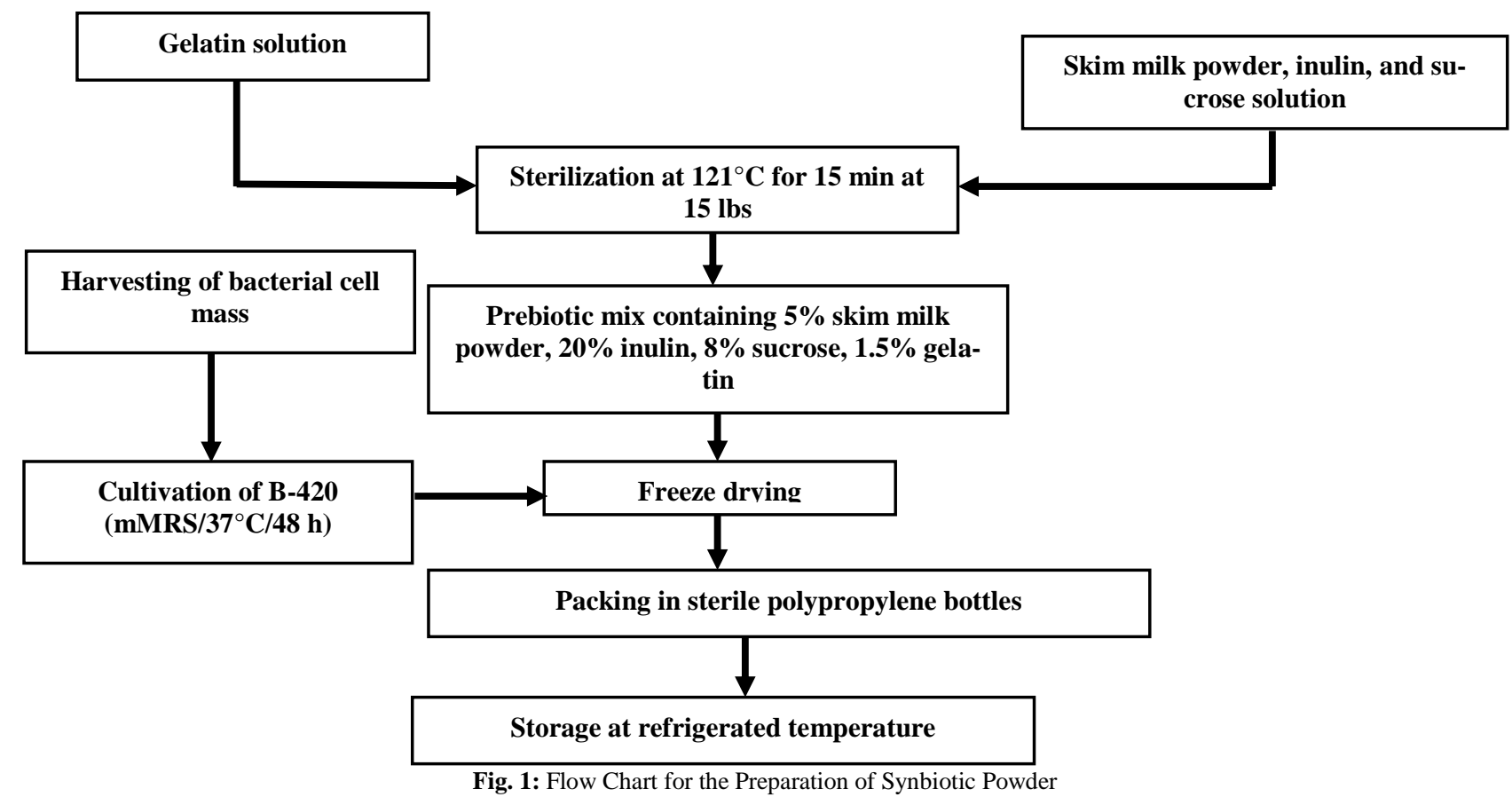


The developed product had a probiotic count of $6.5 \times 10^{8} / \mathrm{g}$ showing a probiotic survival rate of $65 \%$. The probiotic count during room and refrigerated temperatures of storage is depicted in Fig. 2. A marked reduction in the probiotic count (reduction by $3.5 \operatorname{logs}$ ) was observed after 60 days of room temperature storage. No significant difference was observed in the count of the probiotic under refrigerated storage of product. Under refrigeration the count remained in the range of 8 to $9 \log \mathrm{cfu} / \mathrm{g}$ even after 60 days. Via- bility of the probiotic culture during storage is identified as the most important quality indicator of a probiotic product. Contrary to the observation of maintenance of probiotic viability during refrigerated storage there was a marked reduction on room temperature storage with the counts reducing to the level of $<10^{5}$ $\mathrm{cfu} / \mathrm{g}$, highlighting the impact of storage temperature on probiotic viability.

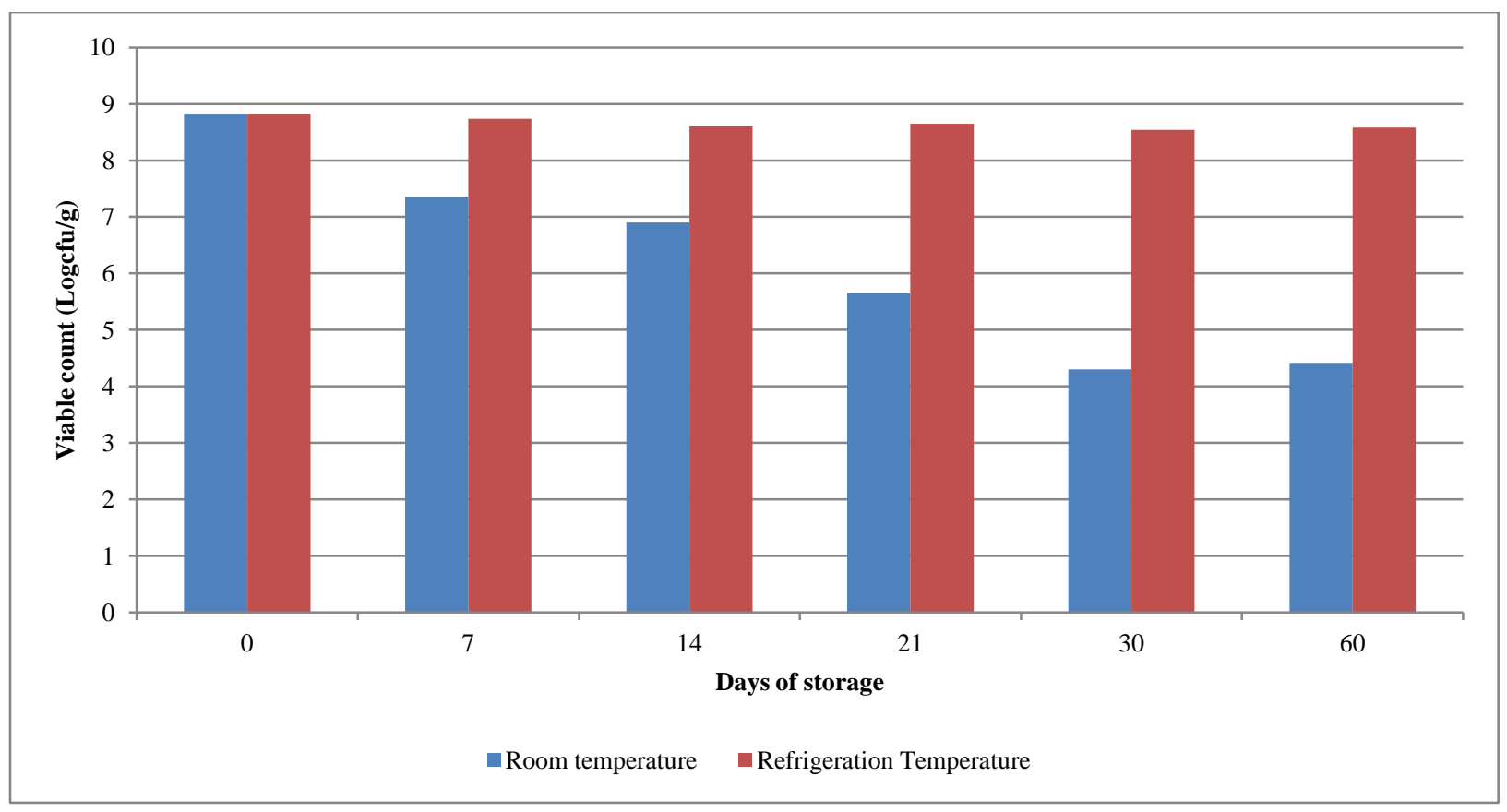

Fig. 2: Viability of the Probiotic Organism (B-420) During Storage of Synbiotic Powder at Room and Refrigeration Temperatures

\section{Discussion}

As synbiotics are synergistic combinations of probiotic bacteria and the unabsorbed substrates they metabolize, the synergistic relationship between these two constituents is essential for developing effective synbiotic products. The capability of the probiotic strain to metabolize fructans as the sole carbon source is considered as an essential pre-requisite while formulating fructancontaining synbiotics (Schrezenmeir and de Vrese, 2001). The ability of a bifidobacterial strain to grow on inulin as the sole carbon source is attributed to the possession of extracellular enzymes that hydrolyze long-chain fructans (Rossi et al. 2005). As both the strains used in this study could utilize inulin, it could be suggested that both of them possess these types of extracellular enzymes. However a marked difference was observed between the strains in their inulin utilization pattern. The strain Bifidobacterium animalis subsp. lactis B420 could grow on inulin at a faster rate than the other strain. Differences in the fermentation rate of inulintype fructans by bifidobacteria is attributed to the variations in their fructan degradation mechanisms (Janer et al. 2004). It could be presumed that the faster growth of Bifidobacterium animalis subsp. lactis B420 on inulin could be advantageous for this strain in terms of survival and proliferation in a highly competitive ecosystem such as the human colon (Flint et al. 2007).Although freeze drying is widely used for preparation of probiotic products, the maintenance of viability of the probiotic during freeze drying is a major hurdle. On assessing the survival rate of Bifidobacterium animalis subsp. lactis B420 in the developed product, $65 \%$ of the probiotic population was found to survive the freeze drying process. Wide variations are reported in the percentage survival of probiotics during freeze drying. Leja et al., (2009) reported a survival rate of $29 \%$ for Lactobacillus rhamonosus GG in a freeze-dried milk preparation, whereas a survival rate as high as $95 \%$ is being reported by Capela et al. (2006). Wang et al. (2004) reported a survival percent of 43.2-51.9\% for bifidobacteria after freeze-drying. It is suggested that the variations observed in the survival rate of microorganisms during spray drying could be attributed to the differences in their temperature resistance (Ananta et al. 2005). Likewise resistance towards freezing temperature, osmotic stress etc. could be contributing towards the survival of microorganisms subjected to freeze drying. The viability of probiotic bacteria during storage is found to be dependent on a number of factors like level of oxygen in the product, storage time and temperature (Shah, 2001). A daily intake of at least $10^{8}-10^{9}$ viable cells is generally considered as the minimum intake to provide a therapeutic effect (Silva et al. 2004). In this context consumption of one gram of the developed product is sufficient to impart a therapeutic effect. Loss of viability of probiotics during the fermentation process and refrigerated storage is identified as a major issue in the production of fermented probiotic products. It is being reported that the viable cells of bifidobacteria in yoghurt-like product could not be maintained in sufficient amounts $\left(>10^{6} \mathrm{cfu} / \mathrm{g}\right)$ for more than 1 week during storage at $4{ }^{\circ} \mathrm{C}$ (Roy et al. 1997, Lamoureux et al. 2002). Based on the results obtained from the storage study, it is quite clear that the developed product served as a good carrier of probiotic organism even after 60 days of storage at refrigeration temperature. So it could be suggested that development of such products will be an attractive alternative to counter the issues like loss of probiotic viability and stability pertaining with the fermented milk products. Inulin, the prebiotic due to its high degree of polymerization (DP) is expected to provide a long lasting effect throughout the colon resulting in the beneficial saccharolytic metabolism and a lowered $\mathrm{pH}$ extended to the distal colon (Rossi et al. 2005). Presence of this prebiotic in the developed product warranties better protection of the probiotic during gastric transit and also provides an upper hand due to the ready availability of a substrate in the gastrointestinal milieu where the competition for nutrients is intense. It has been 
reported that presence of a carbohydrate such as fructans that can be assimilated and metabolized by the probiotic improves the survivability of the probiotic under acidic conditions (Corcoran et al. 2005).

\section{Conclusion}

A synbiotic product was developed by freeze drying a milk based medium containing inulin and Bifidobacterium animalis subsp. lactis B420, a strain selected based on its inulin utilization potential. The developed product was found to retain the viability of probiotic cultures during freeze drying and refrigerated storage, yielding a probiotic count in the range of 8 to $9 \log \mathrm{cfu} / \mathrm{g}$, satisfying the requirement of a bifidobacterial level of 5 to $7 \mathrm{log} \mathrm{cfu} / \mathrm{g}$ considered necessary to derive the probiotic benefit. Being available in a convenient powder form, the developed synbiotic powder could be consumed as such or after reconstitution in a suitable medium. As the next step, it is possible to exploit the possibilities of converting this preparation into a capsular or tablet form for oral administration as a therapeutic agent. An additional area to be investigated is the use of techniques like microencapsulation for enhancing the probiotic stability of the developed product on room temperature storage. This area is of great potential considering the changing trend of application of probiotic cultures in cereal products, drinks and confectionaries which are stored at room temperatures.

\section{Acknowledgements}

Financial assistance in the form of ICAR and CSIR SRF is duly acknowledged.

\section{References}

[1] Ananta E, Volkert M \& Knorr D (2005) Cellular injuries and storage stability of spray-dried Lactobacillus rhamnosus GG. International $\begin{array}{llr}\text { Dairy Journal } & 15, & 399-409\end{array}$ http://dx.doi.org/10.1016/j.idairyj.2004.08.004.

[2] Arroyo, L, Cotton LN \& Martin JH (1994) Evaluation of media for enumeration of Bifidobacterium adolescentis, B. infantis and B longum from pure culture. Cultured Dairy products Journal 29, 20-21, and 23-24.

[3] Bielecka M, Biedrycka E \& Majkowska A (2002) Selection of probiotics and prebiotics for synbiotics and confirmation of their in vivo effectiveness. Food Research International 35, 125-131. http://dx.doi.org/10.1016/S0963-9969 (01)00173-9.

[4] Capela P, Hay TKC \& Shah NP (2006) Effect of cryoprotectants, prebiotics and microencapsulation on survival of probiotic organisms in yoghurt and freeze-dried yoghurt. Food Research International 39, 203-211. http://dx.doi.org/10.1016/j.foodres.2005.07.007.

[5] Collins EB \& Hall BJ (1984) Growth of bifidobacteria in milk and preparation of Bifidobacterium infantis for a dietary adjunct. Journal of Dairy Science 67, 1376-1380. http://dx.doi.org/10.3168/jds.S0022$0302(84) 81451-4$

[6] Corcoran BM, Stanton C, Fitzgerald, GF \& Ross RP (2005) Survival of probiotic lactobacilli in acidic environments is enhanced in the presence of metabolizable sugars. Applied and Environmental Microbiology 71, 3060-7. http://dx.doi.org/10.1128/AEM.71.6.30603067.2005

[7] Desmond C, Ross RP, O'Callaghan E, Fitzgerald G \& Stanton C (2002) Improved survival of Lactobacillus paracasei NFBC 338 in spray-dried powders containing gum acacia. Journal of Applied Microbiology 93, 1003-1011. http://dx.doi.org/10.1046/j.13652672.2002.01782.x

[8] Flint HJ, Duncan SH, Scott KP \& Louis P (2007) Interactions and competition within the microbial community of the human colon: links between diet and health. Environmental Microbiology 9, 11011111. http://dx.doi.org/10.1111/j.1462-2920.2007.01281.x.

[9] Gardiner GE, Bouchier P, O'Sullivan E, Kelly J, Collins JK, Fitzgerald G, Ross RP \& Stanton C (2002) A spray-dried culture for probiotic Cheddar cheese manufacture. International Dairy Journal 12 , 749-756. http://dx.doi.org/10.1016/S0958-6946 (02)00072-9.

[10]Granato D, Branco GF, Nazzaro F, Cruz AG \& Faria JAF (2010) Functional Foods and Nondairy Probiotic Food Development: Trends,
Concepts, and Products. Comprehensive reviews in food science and food safety 9,293-302.

[11]Janer C, Rohr LM, Pelaez C, Laloi M, Cleusix, V, Requena T \& Meile L (2004) Hydrolysis of oligofructoses by the recombinant $\beta$ fructofuranosidase from Bifidobacterium lactis. Systmatic and Applied Microbiology 27,279-285. http://dx.doi.org/10.1078/0723-202000274.

[12]Lamoureux L, Roy D \& Gauthier SF (2002) Production of oligosaccharides in yogurt containing bifidobacteria and yogurt cultures, Journal of Dairy Science 5, 1058-1069. http://dx.doi.org/10.3168/jds.S0022-0302(02)74166-0

[13]Leja K, Dembczyński R, Białas W\& Jankowski T (2009) Production of dry Lactobacillus rhamnosus GG preparations by spray drying and lyophilization in aqueous two-phase systems. ACTA Scientiarum Polonorum Technologia Alimentaria 8, 39-49.

[14]Ma"tto J, Malinen E, Suihko ML, Alander M, Palva A\&Saarela M. (2004) Genetic heterogeneity and technological properties of intestinal bifidobacteria. Journal of Applied Microbiology 97, 459-470. http://dx.doi.org/10.1111/j.1365-2672.2004.02340.x.

[15]Palaria A, Johnson-Kanda I\& O'Sullivan D J (2012) Effect of a Synbiotic Yogurt on Levels of Fecal Bifidobacteria, Clostridia, and Enterobacteria. Applied and Environmental Microbiology 78, 933-940. http://dx.doi.org/10.1128/AEM.05848-11.

[16]Rasic J (1990) Culture media for detection and enumeration of the bifidobacteria in fermented milk products. Bull.etin of the International Dairy Federation 252, 24-31.

[17]Rossi M, Corradini C, Amaretti A, Nicolini M, Pompei A, Zanoni S \& Matteuzzi D (2005) Fermentation of fructooligosaccharides and inulin by bifidobacteria: a comparative study of pure and fecal cultures. Applied and Environmental Microbiology 71, 6150-6158. http://dx.doi.org/10.1128/AEM.71.10.6150-6158.2005.

[18]Roy D, Mainville I \& Mondou F (1997) Bifidobacteria and their role in yogurt-related product. Microecology and Therapy 26, 167-180.

[19]Saulnier DMA, Gibson GR\& Kolida S (2008) In vitro effects of selected synbiotics on the faecal microbiota composition. FEMS Microbiology Ecology 66, 516-527. http://dx.doi.org/10.1111/j.15746941.2008.00561.x

[20]Schrezenmeir J \& de Vrese M (2001) Probiotics, prebiotics, and synbiotics approaching a definition. American Journal of Clinical Nutrition 73(suppl), 361S-4S.

[21] Shah NP (2001) Functional Foods from probiotics and prebiotics. Food Technology 55, 46-53.

[22]Silva IS, Barbosa FH, Duarte R, Vieira LQ, Arantes RM \& Nicoli JR (2004) Effect of Bifidobacterium longum ingestion on experimental salmonellosis in mice, Journal of Applied Microbiology 97, 29-37. http://dx.doi.org/10.1111/j.1365-2672.2004.02265.x.

[23] Wang Y, Yu C \& Chou C (2004) Viability of lactic acid bacteria and bifidobacteria in fermented soymilk after drying, subsequent rehydration and storage. International Journal of Food Microbiology 93, 209. 217. http://dx.doi.org/10.1016/j.ijfoodmicro.2003.12.001. 